OPEN ACCESS

Edited by:

Gary Cheung,

The University of Auckland,

New Zealand

Reviewed by:

Hayriye Gulec,

Uludag University, Turkey

Marco Matthaeus Zierhut,

Charité - Universitätsmedizin

Berlin, Germany

*Correspondence:

Lynn Martin

lynn.martin@lakeheadu.ca

Specialty section:

This article was submitted to

Public Mental Health,

a section of the journal

Frontiers in Psychiatry

Received: 26 February 2021

Accepted: 26 April 2021

Published: 28 May 2021

Citation:

Herring MG, Martin L and Kristman VL (2021) Brief Report: Characteristics and Needs of Persons Admitted to an Inpatient Psychiatric Hospital With Workers' Compensation Coverage.

Front. Psychiatry 12:673123.

doi: 10.3389/fpsyt.2021.673123

\section{Brief Report: Characteristics and Needs of Persons Admitted to an Inpatient Psychiatric Hospital With Workers' Compensation Coverage}

\author{
Mary Grace Herring ${ }^{1,2}$, Lynn Martin ${ }^{1,2 *}$ and Vicki L. Kristman ${ }^{1,2}$ \\ ${ }^{1}$ Department of Health Sciences, Lakehead University, Thunder Bay, ON, Canada, ${ }^{2}$ Enhancing Prevention of Injury \& \\ Disability (EPID)@Work Research Institute, Lakehead University, Thunder Bay, ON, Canada
}

The rise of mental health issues in the workplace is widely known. Though mental health issues were not covered by the Workplace Safety Insurance Board (WSIB) in Ontario (Canada) until 2018, it was listed as responsible for payment of inpatient psychiatric hospital stays between 2006 and 2016. This population-level observational analytic study compares the clinical and service needs of 1,091 individuals admitted to inpatient psychiatry with WSIB coverage to all other admissions $(n=449,128)$. Secondary analysis was based on the interRAI Mental Health assessment. The WSIB group differed from all other admissions on almost all characteristics considered. Most notably, depression (65.08 vs. $57.02 \%$ ), traumatic life events (25.48 vs. $15.58 \%$ ), substance use (58.02 vs. $46.92 \%)$, daily pain (38.31 vs. $12.15 \%$ ) and sleep disturbance (48.95 vs. $37.12 \%$ ) were much higher in the WSIB group. Females with WSIB coverage had more depression (74.36 vs. $59.91 \%$ ) and traumatic life events (30.00 vs. $22.97 \%)$, whereas males had more substance issues (63.62 vs. 47.95\%). In addition, persons under the age of 55 had more substance issues ( $<25=75.47 \%$; $25-54=61.64 \%$ : $55 \pm 40.54 \%$ ) and traumatic life events ( $<25=26.41 \%$; $25-54=28.18 \%$; $55 \pm 15.31 \%)$, while those $25-54$ years had more daily pain (41.67\% vs. $<25=3.77 \%$ and $55 \pm 34.23 \%$ ) and sleep disturbance (50.74\% vs. $<25=33.96 \%$ and $55 \pm 45.94 \%$ ). All variables differed significantly by sex and age within the comparison group, though not always following the patterns observed in the WSIB group. Future research examining mental health needs and outcomes among injured workers receiving inpatient psychiatric services is needed, and should take into account sex and age.

Keywords: interRAl, mental health, inpatient psychiatry, worker, trauma, pain, substance use, depression

\section{INTRODUCTION}

That mental health conditions are on the rise is widely known. These affect hundreds of millions of people internationally, and are now recognized among the leading causes of disability (1). By 2030, the global cost of mental illness will surpass six trillion dollars (2). In Canada, the annual economic burden of mental illness is $\sim 50$ billion dollars, and is projected to reach 307 billion by 2041 (2).

The rise of mental health issues in the workplace is equally widely known. A systematic review and meta-analysis found that $\sim 18 \%$ of workers met the criteria for common mental health 
conditions (i.e., mood, anxiety, substance-related disorders), with a lifetime prevalence of just under $30 \%$ (3). It is not surprising then, that programs and policies targeting mental health in the workplace have become a focus for employers and governments around the world.

In Canada, workers' compensation started with the 1913 Meredith Report; it outlined an arrangement in which workers ceded their right to sue in return for benefits (4). The main tenets of the original workers' compensation laws still exist: no-fault compensation, collective liability, security of payment, exclusive jurisdiction, and independent board. In spite of having common principles, Canadian provinces and territories have their own Workers' Compensation Board (WCB), apart from the Northwest Territories and Nunavut, which share a WCB. Governments in each jurisdiction create Workers Compensation legislation, which is administered by the WCB. The benefits provided to workers most commonly fall into one of five categories: health care, wage loss benefits, permanent disability benefits, fatal and dependency benefits (survivor benefits), and rehabilitation. The levels of coverage and benefit amounts vary by WCB. For example, the percentage of earnings that benefits are based on vary: 85\% net in Prince Edward Island and Ontario, and 90\% net in Quebec, Manitoba, Saskatchewan, Alberta, British Columbia, and Northwest Territories and Nunavut. Federally, all government employees are governed under the Federal Government Employees Compensation Act.

While for some, mental health conditions may not originate in the workplace, there is considerable evidence that the workplace may itself contribute to mental illness (5). This led to changes in Ontario's Workplace Safety and Insurance Board (WSIB); its new chronic mental stress policy and revised traumatic mental stress policy came into effect on January 1, 2018. Ontario's WSIB previously compensated chronic mental health injuries until Bill 99 removed this coverage in 1998. In 2016, Bill 163 was passed that recognized posttraumatic stress disorder in first responders to be a workrelated injury, unless proven otherwise. However, there was still no coverage for mental health injuries developed over time. In 2017, the Ontario government passed Bill 177, which amended the Act to cover chronic and traumatic mental stress as long as the stress is not caused by "decisions or actions of the worker's employer relating to the worker's employment, including a decision to change the work to be performed or the working conditions, to discipline the worker or to terminate the employment" (6).

Though mental health issues were not explicitly covered by the WSIB until 2018, it was listed as responsible for payment of inpatient psychiatric stays between January 1, 2006 and December 31, 2016. This study compares the clinical and service needs of the 1,091 individuals admitted to inpatient psychiatry in this 11-year period to all other admissions. Given that the nature and experience of work-related injuries and disabilities are known to differ by sex and age $(7,8)$, within and between-group comparisons by age and sex will be made.

\section{MATERIALS AND METHODS}

\section{Data}

This observational analytic study employed secondary analysis of anonymized population-level clinical data in the Ontario Mental Health Reporting System (OMHRS) collected between January 1, 2006 and December 31, 2016 on all persons admitted to an adult inpatient psychiatric bed or unit. Anonymized data are held on a secure server at the University of Waterloo as part of a datasharing agreement between the Ontario Ministry of Health and interRAI, a not-for-profit research consortium. Exemption from review for secondary analysis of anonymized data was granted by the Lakehead University Research Ethics Board, as per the Tri-Council Policy Statement (9).

\section{Instrument}

Data are based on the RAI Mental Health assessment (10-12), which contains over 300 items targeting key domains: personal information, mental health service history, mental state, substance use, injurious behaviors, social roles and relationships, psychiatric diagnoses, cognition, functioning, health conditions and medical diagnoses, medications, life stressors, service use, and informal supports. Items are also grouped into various scales, algorithms, and protocols framed on recovery principles that support evidence-informed individual-level care planning and shared decision-making processes about services $(12,13)$. It is completed at admission and discharge (if the stay lasts at least 7 days); reassessments are completed every 90 days if still in hospital.

\section{Study Population/Variables}

Admission assessments were completed on 449,128 unique individuals in the study period; if individuals had more than one admission, the most recent assessment was used. The group of interest are workers who experience mental health issues, defined as those for whom WCB/WSIB was listed as a source responsible for payment. The comparison group represents all other admissions.

Items related to personal information (i.e., age, sex, marital status, living arrangements) and stay (i.e., reasons for admission, length of stay) were used, as were DSM-V diagnostic categories (actual or provisional diagnoses). All embedded scales available in the assessment were used to describe clinical characteristics: Activities of Daily Living Hierarchy (ADLH) (14), Aggressive Behavior Scale (ABS) (15), Cognitive Performance Scale (CPS) (16), Depression Rating Scale (DRS) (17), substance use (CAGE scale) (12), Mania Scale 10, Positive Symptoms Scale (12), Social Withdrawal Scale (SWS, formerly called Negative Symptoms Scale, NSS) (12), and Pain Scale (18). Previouslyestablished cut-offs were used for each scale to indicate problems in that area (e.g., higher severity, frequency). A succinct description of the items and coding used in embedded scales is available elsewhere (19).

Just as scales represent the combination of items to provide information on overall status in different areas, items are also combined to flag issues. These are called clinical assessment 
TABLE 1 | Number of WSIB admissions between January 1, 2006 and December 31, 2016.

\begin{tabular}{lcc}
\hline Year & Total admissions $(\boldsymbol{N})$ & WCB/WSIB N (\% of all admissions) \\
\hline 2006 & 38,655 & $103(0.27 \%)$ \\
2007 & 38,984 & $88(0.23 \%)$ \\
2008 & 38,399 & $90(0.23 \%)$ \\
2009 & 39,426 & $83(0.21 \%)$ \\
2010 & 40,206 & $56(0.14 \%)$ \\
2011 & 40,636 & $95(0.23 \%)$ \\
2012 & 41,551 & $104(0.25 \%)$ \\
2013 & 41,490 & $107(0.26 \%)$ \\
2014 & 42,432 & $109(0.26 \%)$ \\
2015 & 43,110 & $127(0.29 \%)$ \\
2016 & 44,239 & $129(0.29 \%)$ \\
Total & 449,128 & $1,091(0.24 \%)$ \\
\hline
\end{tabular}

protocols (CAPs), and identify needs related to safety (i.e., harm to self and others, suicidality and purposeful selfharm, self-care), social life (i.e., social relationships, informal support, support systems for discharge, interpersonal conflict, traumatic life events, criminal activity), economic issues (i.e., personal finance, education, and employment), autonomy (i.e., control interventions, medication management and adherence, rehospitalization), and health promotion (i.e., smoking, substance use, weight management, exercise, sleep disturbance, pain, falls) $(12,13,20)$.

\section{Analysis}

The prevalence of persons admitted with WSIB coverage is shown annually and overall. Descriptive statistics (\%, mean, standard deviation) inform on all variables, and relevant tests of significance were used to report on differences within and between groups (chi-square, $t$-test, ANOVA). Given the very large number of admission assessments, a strict Type I error rate was used (i.e., alpha $=0.001$ ); only findings that meet this criteria are described. The same reporting format is used for all results: (a) description of between group differences, (b) description of within-WSIB group differences by age and sex, and (c) description of within-Other group differences by age and sex that differ from what was found in the WSIB group. As such, within-Other group differences by sex and age that are not described are similar to those within the WSIB group.

\section{RESULTS}

There were 1,091 assessments that had WSIB listed among responsible sources of payment, representing $0.24 \%$ of all admissions (Table 1). Such admissions ranged between 56 and 129 per year; they decreased between 2006 and 2010 and then steadily increased.

\section{Study Population Characteristics}

Table 2 shows personal and admission characteristics; multiple reasons may be listed for each admission.

\section{Between Group Differences}

The WSIB mean age was higher $[45.37$ years $(S D=12.4)$ vs. 42.91 years $(\mathrm{SD}=17.0) ; p>0.001]$ than the other group. The majority of people in both groups were male (WSIB: 64.25\%; $p<0.001$; Other: $50.99 \%$; $p<0.001$ ), equal proportions lived alone and the majority lived in a private home. However, more people in the WSIB group were married, lived with a spouse and other(s), whereas more in the other group had never been married, lived with non-relatives or other relatives, and had been admitted from an acute or other setting. The WSIB group was less often admitted for all reasons listed, with one exception: this group had more admissions due to problems with addiction or dependency. The mean length of stay was about 4 days, and did not significantly differ between groups [WSIB: 3.89 days ( $\mathrm{SD}=10.9)$; Other: 4.05 days $(\mathrm{SD}=18.9) ; p=0.77]$.

\section{Within-WSIB Group Differences by Sex and Age}

Within the WSIB group, the youngest group (i.e., under 25 years) tended to have never been married, whereas most in the oldest group (i.e., 55 years or more) were married; there were equal proportions among those 25-54 years who had never been married and who were currently married. Females more often lived with a child only compared to males, the youngest with other relatives (e.g., parent or guardian), and the two older groups most often lived alone. Females were more likely to be admitted due to threat or danger to self, inability to care for self, and specific psychiatric symptoms. Males were more often admitted due to problems with addiction or dependency and involvement with the criminal justice system. The youngest group was most often admitted for being a danger to others, while admission due to inability to care for self was highest among the oldest group. Admission for specific psychiatric symptoms was most frequent among those aged 25 to 54 years; the proportion admitted for addiction and dependency was similar among those under 25 years and between 25 and 54 years. There were no sex differences for mean age $(p=0.09)$, marital status $(p=0.16)$, place admitted from $(p=0.22)$, admission for threat/danger to others $(p=0.06)$, and mean length of stay $(p=0.58)$. There were no age differences for admission due to threat or danger to self $(p=0.07)$, justice system/forensic $(p=0.69)$, other reasons $(p=0.26)$, and mean length of stay $(p=0.22)$.

\section{Within-Other Group Differences by Sex and Age}

Some notable differences existed by sex and age in the other group. Compared to males, females were older, and more commonly lived with their spouse and children. They were also more often admitted from a private home, and fewer had been homeless. Males were more often admitted for being a danger or threat to others. Unlike the WSIB group, there were agerelated differences for all reasons for admission. In particular, the youngest group was most often admitted for threat or danger to self and involvement with the justice or forensic system, while those 25-54 years were more often admitted due to inability to care for self. For their part, the oldest group was most often admitted due to specific psychiatric symptoms and for reasons other than those listed. The mean length of stay was statistically significantly higher among males and in the oldest age group, 
TABLE 2 | Univariate distribution (\%) of personal characteristics of persons admitted overall, and by WSIB status, sex, and age.

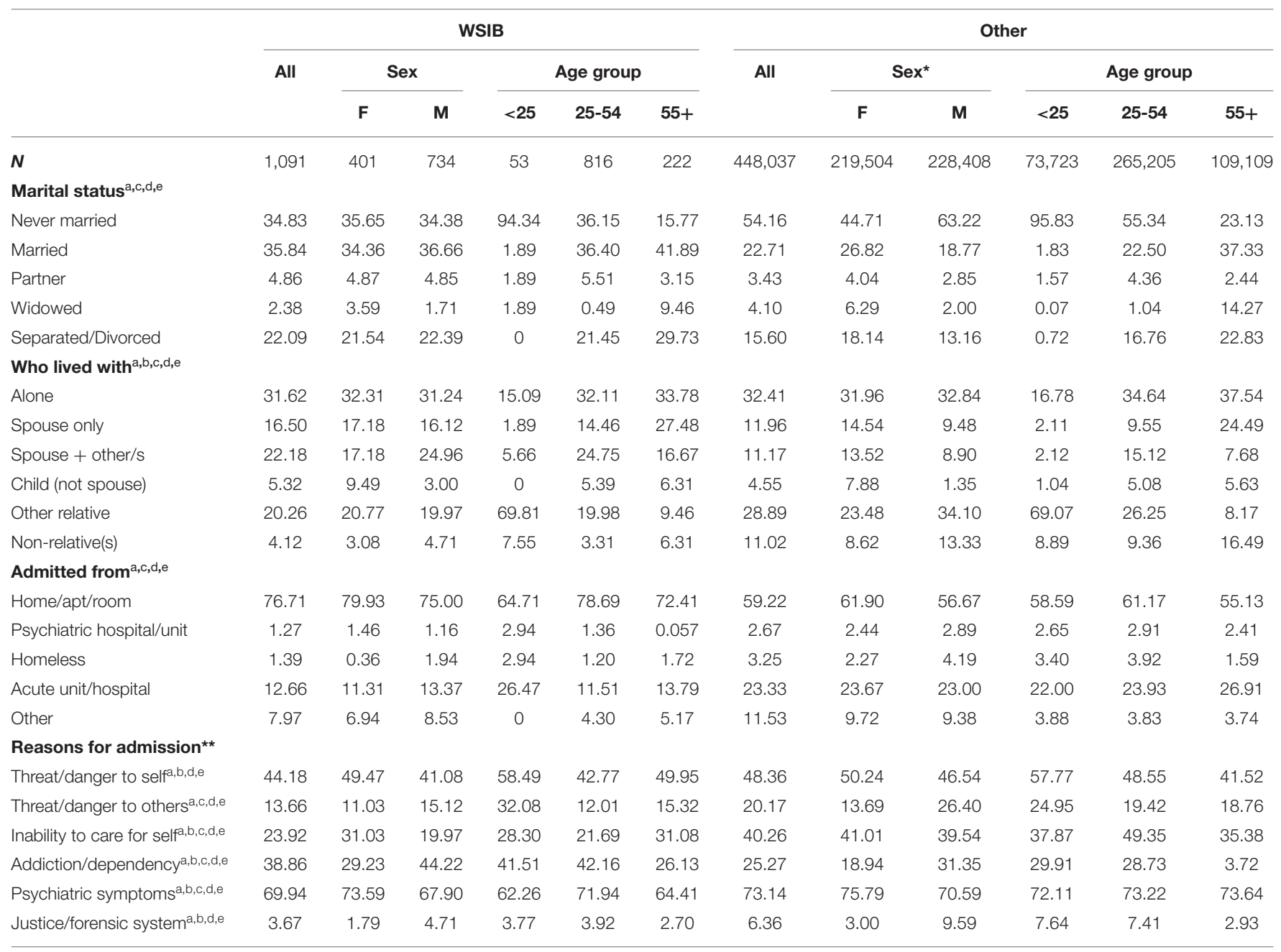

"An additional N = 125 identified their sex as "other"; "Multiple reasons may be listed for each admission; a Significant difference between the WSIB and Other group ( $p<0.001)$; ${ }^{b}$ Significant difference by sex within the WSIB group $(p<0.001)$; ${ }^{\circ}$ Significant difference by age category within the WSIB group $(p<0.001) ;{ }^{d}$ Significant difference by sex within the Other group ( $p<0.001)$; e Significant difference by age category within the Other group $(p<0.001)$.

though the actual differences were minimal [4.16 days (SD = $22.9)$ vs. 3.95 days $(S D=13.6)$ among females; 4.33 days $(S D=$ $24.5)$ vs 3.97 days $(\mathrm{SD}=17.2)$ among $25-54$ years and 4.00 days $(\mathrm{SD}=22.9)$ among under 25 years $]$.

\section{Clinical Characteristics}

Table 3 reports on DSM-V diagnostic categories and clinical characteristics as measured by embedded scale scores.

\section{Between Group Differences}

Differences existed between the two groups for all but personality disorder diagnosis $(p=0.02)$, mania $(p=0.17)$, social withdrawal $(p=0.10)$, and ADL impairment $(p=0.09)$. A higher proportion of those in the WSIB group had substance-related, mood, and anxiety disorders, whereas schizophrenia/psychotic disorders were more prevalent in the other group. More persons in the WSIB group exceeded the cut-off scores for depression, possible substance problem, and pain; moderate or worse cognitive impairment, severe aggression, and positive symptoms were more common in the other group.

\section{Within-WSIB Group Differences by Sex and Age}

In the WSIB group, more females had a mood disorder diagnosis and exceeded the cut-offs for depression and social withdrawal, whereas more males had a substance-related diagnosis and exceeded the cut-off for possible substance problem. There were no sex differences for the other diagnostic categories (schizophrenia/psychotic: $p=0.39$; anxiety: $p=0.70$; personality: $p=0.08$ ) and scales (CPS: $p=0.73$; ADLH: $p=0.86$; ABS: $p=0.09$; Mania: $p=0.14$; PSS: $p=0.49$; Pain: $p=0.33$ ). More persons in the youngest group had schizophrenia/psychotic disorder, severe aggression, possible substance problem, and positive symptoms, while more in the 25-54 years group had diagnoses related to substances, mood, and anxiety disorders and exhibited signs of daily pain. The oldest most often had moderate or worse cognitive and ADL impairment. Age differences did not 
TABLE 3 | Univariate distribution (\%) for clinical characteristics overall, and by WSIB status, sex, and age.

\begin{tabular}{|c|c|c|c|c|c|c|c|c|c|c|c|c|}
\hline & \multicolumn{6}{|c|}{ WSIB } & \multicolumn{6}{|c|}{ Other } \\
\hline & \multirow[t]{2}{*}{ All } & \multicolumn{2}{|c|}{ Sex } & \multicolumn{3}{|c|}{ Age category } & \multirow[t]{2}{*}{ All } & \multicolumn{2}{|c|}{$\operatorname{Sex}^{\star}$} & \multicolumn{3}{|c|}{ Age category } \\
\hline & & $\mathbf{F}$ & $\mathbf{M}$ & $<25$ & $25-54$ & $55+$ & & $\mathbf{F}$ & $\mathbf{M}$ & $<25$ & $25-54$ & $55+$ \\
\hline$N$ & 1,091 & 401 & 734 & 53 & 816 & 222 & 448,037 & 219,504 & 228,408 & 73,723 & 265,205 & 109,109 \\
\hline \multicolumn{13}{|l|}{ DSM-IV categories } \\
\hline Substance-related ${ }^{a, b, c, d, e}$ & 36.66 & 25.90 & 42.65 & 16.98 & 40.93 & 25.68 & 24.90 & 17.73 & 31.79 & 29.59 & 28.38 & 13.29 \\
\hline Schizophrenia/psychotic ${ }^{a, c, d, e}$ & 16.41 & 15.13 & 17.12 & 35.85 & 15.32 & 15.77 & 36.27 & 29.80 & 42.48 & 39.30 & 38.66 & 28.38 \\
\hline $\operatorname{Mood}^{a, b, c, d, e}$ & 60.49 & 71.28 & 54.49 & 43.40 & 62.62 & 56.76 & 50.81 & 59.10 & 42.85 & 45.78 & 50.18 & 55.77 \\
\hline Anxiety ${ }^{a, c, d, e}$ & 31.07 & 31.79 & 30.67 & 24.53 & 34.93 & 18.47 & 12.87 & 15.54 & 10.29 & 12.85 & 13.30 & 11.83 \\
\hline Personality ${ }^{d, e}$ & 8.07 & 10.00 & 6.99 & 11.32 & 8.58 & 5.41 & 10.13 & 12.76 & 7.60 & 13.29 & 11.10 & 5.63 \\
\hline \multicolumn{13}{|l|}{ Other $^{* \star}$} \\
\hline Moderate or worse cognitive impairment (CPS $3+)^{a, c, d, e}$ & 4.12 & 3.85 & 4.28 & 7.55 & 2.70 & 8.56 & 8.27 & 8.09 & 8.45 & 5.04 & 5.00 & 18.42 \\
\hline Moderate or worse ADL impairment ADLH $3+)^{3,4,5}$ & 4.22 & 4.36 & 4.14 & 1.89 & 3.09 & 9.01 & 5.38 & 5.53 & 5.25 & 2.18 & 2.64 & 14.22 \\
\hline Severe aggression (ABS 5+) ${ }^{a, c, d, e}$ & 4.49 & 5.90 & 3.71 & 9.43 & 3.55 & 6.76 & 9.48 & 8.90 & 10.05 & 10.74 & 8.35 & 11.40 \\
\hline Possible depression (DRS 3+) $)^{1,2,4,5}$ & 65.08 & 74.36 & 59.91 & 54.72 & 66.91 & 60.81 & 57.02 & 65.01 & 49.34 & 54.86 & 56.65 & 59.38 \\
\hline Possible substance problem (CAGE 2+) a,b,c,d,e & 25.30 & 15.90 & 30.53 & 28.30 & 27.82 & 15.32 & 17.79 & 13.66 & 21.76 & 19.55 & 20.64 & 9.69 \\
\hline Any sign of mania (Mania Scale $1+)^{\mathrm{d}, \mathrm{e}}$ & 54.72 & 57.69 & 53.07 & 62.26 & 54.66 & 53.15 & 56.76 & 56.54 & 56.98 & 59.76 & 56.61 & 55.10 \\
\hline Any positive symptom (PSS 1+) ${ }^{a, c, d, e}$ & 28.23 & 29.49 & 27.53 & 54.72 & 25.61 & 31.53 & 48.61 & 45.46 & 51.65 & 50.77 & 48.60 & 47.20 \\
\hline Any social withdrawal (SWS $1+)^{\mathrm{b}, \mathrm{d}, \mathrm{e}}$ & 61.69 & 66.15 & 59.20 & 62.26 & 62.62 & 58.11 & 59.22 & 61.49 & 57.04 & 57.83 & 58.89 & 60.97 \\
\hline Daily pain (Pain Scale $2+)^{a, c, d, e}$ & 38.31 & 36.41 & 39.37 & 3.77 & 41.67 & 34.23 & 12.15 & 13.89 & 10.47 & 4.78 & 12.48 & 16.31 \\
\hline
\end{tabular}

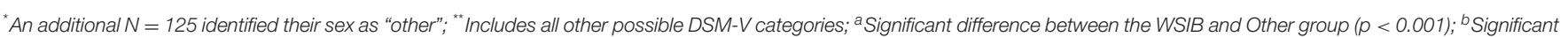

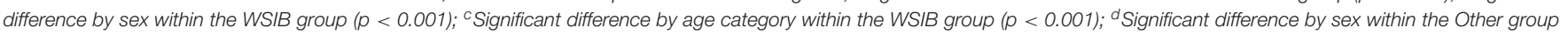
$(p<0.001)$; ' Significant difference by age category within the Other group $(p<0.001)$.

exist for personality disorder diagnosis $(p=0.21)$, depression $(p$ $=0.06)$, social withdrawal $(p=0.47)$, and mania $(p=0.49)$.

\section{Within-Other Group Differences by Sex and Age}

Different patterns of sex and age-related findings were noted in the other group. Males were more often diagnosed with schizophrenia/psychotic disorder and exceeded cut-offs related to cognition, aggression, mania, and positive symptoms, whereas this was true for anxiety and personality disorders, ADL impairment, and pain among females. In this group, the oldest more often had mood disorder diagnoses and exceeded cutoffs for aggression, depression, social withdrawal, and pain, while signs of mania, substance-related disorders, and personality disorders were most common in the youngest. A potential problem with substances was highest for those 25 to 54 years.

\section{Areas of Need}

Table 4 reports on areas of need as measured by CAPs.

\section{Between Group Differences}

CAPs for traumatic life events, substance use, weight management, sleep disturbance, and pain were more often triggered in the WSIB group; harm to others, self-care, supports for discharge, criminal activity, personal finances, education and unemployment, control interventions, medication management and adherence, rehospitalization, and smoking were more common in the comparison group. There were no differences for suicidality and purposeful self-harm $(p=0.06)$, social relationships $(p=0.52)$, interpersonal conflict $(p=0.07)$, exercise $(p=0.71)$, and falls $(p=0.09)$.

\section{Within-WSIB Group Differences by Sex and Age}

Females in the WSIB group more often had needs related to suicidality and purposeful self-harm, social relationships, interpersonal conflict, and traumatic life events. Males more often triggered CAPs for criminal activity, smoking, and substance use. There were no differences by sex for all other CAPs ( $p$-values ranged from 0.07 to 0.84 ). The youngest group more frequently triggered almost all CAPs, including harm to others, suicidality and purposeful self-harm, self-care, criminal activity, personal finances, education and employment, medication management and adherence, rehospitalization, and substance use. Traumatic life events, smoking, sleep disturbance, and pain were most common among those aged 25 to 54 years. There were no age differences for social relationships $(p=0.20)$, support for discharge $(p=0.09)$, interpersonal conflict $(p=0.06)$, control interventions $(p=0.06)$, weight management $(p=0.30)$, exercise $(p=0.26)$, and falls $(p=0.20)$.

\section{Within-Other Group Differences by Sex and Age}

In the other group, the triggering of all CAPs differed by both sex and age. Different than what was found in the WSIB group, females in this group more frequently triggered CAPs for weight management, exercise, sleep disturbance, pain, and falls. Males more often had needs related to harm to others, self-care, supports for discharge, interpersonal conflict, personal finances, education and employment, control 
TABLE 4 | Univariate distribution (\%) for areas of need overall, and by WSIB status, sex, and age.

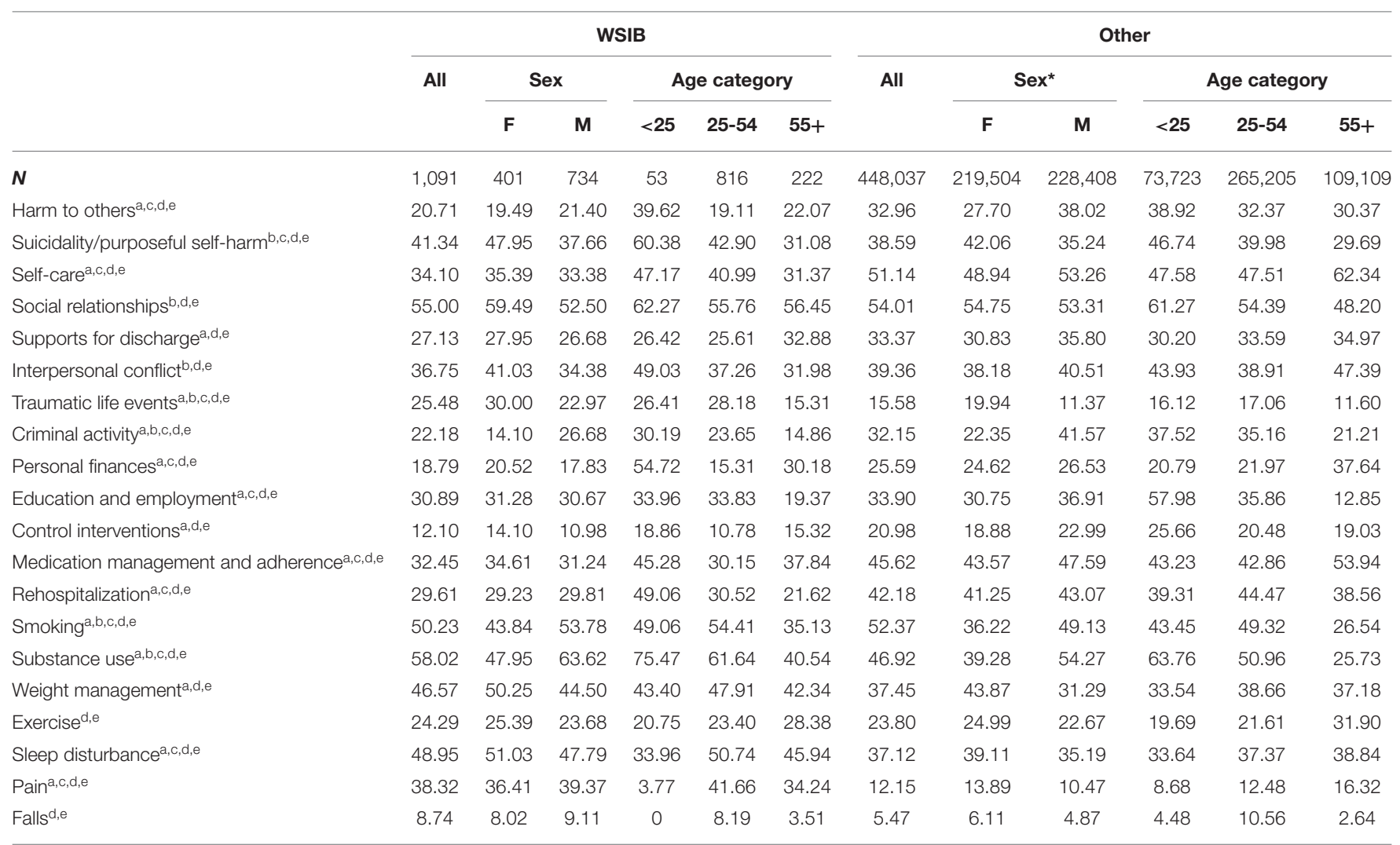

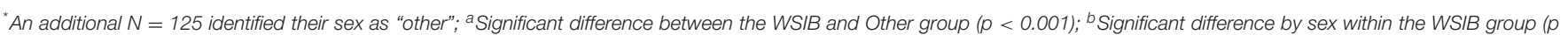
$<0.001) ;{ }^{C}$ Significant difference by age category within the WSIB group $(p<0.001) ;{ }^{d}$ Significant difference by sex within the Other group $(p<0.001)$; ${ }^{e}$ Significant difference by age category within the Other group $(p<0.001)$.

interventions, medication, and rehospitalization. The youngest group had additional needs around social relationships and control interventions, while this was true for rehospitalization, weight management, and falls among those 25-54 years. The oldest more often had needs related to self-care, supports for discharge, interpersonal conflict, personal finances, medication management and adherence, exercise, sleep disturbance, and pain.

\section{DISCUSSION}

This study reports on over a thousand Ontarians whose psychiatric admission was the responsibility-partially or fullyof the WSIB between 2006 and 2016; which is up to 12 years prior to its coverage for chronic mental stress. As such, it is suspected that WSIB was the payor due to a comorbid workplace physical injury or illness, or to severe traumatic experience. Approximately one quarter of individuals in this group had experienced trauma, and presumably, the remainder received coverage related to physical injuries. While those with WSIB coverage represent a small minority of psychiatric admissions, it has been a growing one. As the WSIB expanded coverage to chronic mental stress in 2018, it is expected that the number of admissions will continue to grow. Future studies should examine the profiles of persons admitted since the change in legislation.

In this study, the WSIB group tended to be comprised of males, which is consistent with the distribution of WSIB claims; for example, between 2006 and 2016, males represented $57-64 \%$ of all WSIB claims (21). Similar distributions have been reported in other provinces (22). The age distribution across all WSIB claims within the same time frame, however, is different. While the majority of claims were made by individuals between 40 and 54 years of age in both populations, those in this age bracket represented $40.35 \%$ of all WSIB claims and $49.86 \%$ of the study population. The biggest difference was seen amongst people under the age of 25 , who represented $12.22 \%$ of claims and $4.51 \%$ of the study population (21). In Ontario, longer claim duration was associated with older age (23), and longer unemployment is associated with psychological distress (24). Future studies that use data linkage between WSIB claims and interRAI are needed to better understand the timing of injury, claims, and admissions. This would help to understand whether those in this study were older because they were older at the time of their injury, or because they were off of work for a longer duration and experienced negative mental health consequences because of longer unemployment. 
The personal characteristics of the WSIB group were different than that of the comparison group. There were more people in the WSIB group who were married and living with a spouse than in the group not covered by the WSIB. This may help to explain the finding that fewer people in the WSIB group triggered for potential problems with availability of a support system after discharge, though it is important to note that more than one quarter did. Further, eligibility for coverage by WSIB means that these individuals had been employed, making them less likely to trigger the two CAPs related to economic issues. Again though, it needs to be noted that almost a third of individuals in the WSIB group did have issues related to education and employment, and so return to work-to either previous or new employment, or possibly retraining/education remain areas of concern.

In terms of the clinical characteristics and areas of need, the findings reported in this study are in line with the literature showing increased depression (24-26), substance use (27-29), and pain (30) among those who have experienced workplace injury. The literature has also described the co-occurrence and relationships between workplace injury, pain, depression, and substance use (30). While beyond the scope of this descriptive study, such relationships could easily be examined in the data. For example, subsequent analyses (not shown) revealed that $31.74 \%$ in the WSIB group experienced daily pain, and this increased to $47.88 \%$ among those admitted for addiction or dependency; that about two thirds of males in the WSIB group had needs related to substance use, and this increased to $72.46 \%$ among those with daily pain. That pain, depression, and substance use each represent chronic conditions provide impetus for further exploration of these relationships in the data cross-sectionally and longitudinally. Given that the interRAI instrument also collects information related to service use and is completed at the time of discharge, evaluation of adequate pain management on outcomes and the extent to which it may potentially reduce the likelihood or severity of mental health issues are possible, as is looking at impact on length of stay.

The use of sex- and age-based analyses further describes the specific needs of injured workers admitted to inpatient psychiatry. In particular, that almost three quarters of females in the WSIB group showed signs of clinically relevant depression, as well as more traumatic life events and issues related to social relationships, interpersonal conflict, and suicidality and purposeful self-harm represents a very different profile than males. Many of these needs were also shown to be more prevalent in the youngest group (i.e., under 25 years). More specifically, they had more needs related to safety (suicidality and purposeful self-harm, harm to others, self-care), social life (criminal activity), economic issues (personal finance, education and employment), and autonomy (medication management and adherence, rehospitalization), and displayed more severe aggression, positive symptoms, and issues with substances. While in and of itself, it is not surprising that the youngest group would more often experience some of these issues (e.g., related to education, personal finances), it is somewhat surprising that they are experiencing higher rates of most issues assessed in the instrument. While a recent systematic review examined associations between work-related stressors and the mental health of young workers (31), to our knowledge, the focus of research on younger injured workers remains largely on the physical aspects of the injuries. This study points to the need for additional focus on the mental health needs and outcomes among young injured workers. Further research that examines clinical needs among males and females of different ages is recommended. In addition, longitudinal research is needed on outcomes over time, including rehospitalization, to better understand the factors influencing recovery. That just under half of those under the age of 25 years were at risk for rehospitalization further points to the complexity of issues facing this group and the need to intervene as soon as possible.

The interRAI instrument contains over 300 items on the strengths, preferences, needs, and service use in inpatient psychiatry, but it does not cover work-related disability specifically. As such, it does not provide information on the timing or nature of the work-related injury. Similarly, the instrument assesses for presence of traumatic events, depression, self-harm, substance us, and pain, for example, but does not have specific information on the timing of onset. Intersectoral collaboration is needed to develop mechanisms to link WSIB and inpatient psychiatry data to better understand the mental health needs of injured workers, and to explore the causal pathways involved.

A major strength of this study is the scope of variables included. The instrument's items, scales, and CAPs have demonstrated reliability and validity, and allow researchers to analyze a range of characteristics, thus providing a comprehensive description of the study population. A second strength is the population-based nature of the data. All persons admitted into a psychiatric facility or psychiatric unit in a hospital are required to be assessed with this instrument. Therefore, the entire population of interest is included in this study. A limitation, however, is that there is no way to know why the person was covered by the WSIB, nor when this occurred. They could have had a physical injury or a severe traumatic experience at work that resulted in work disability, which could have happened 5 years ago or 1 year ago. As stated previously, future studies linking WSIB claims and interRAI inpatient psychiatry population data are recommended. This will become increasingly important given the 2018 policy change to include coverage for chronic mental stress. Another drawback includes the inability to assess the timeline of the mental illness that led to the admission of the individual at a psychiatric facility and the injury that resulted in WSIB coverage. It would be interesting to see the effects of time off work on mental health illness, as well as the order of events between work injury and mental health illness.

\section{CONCLUSION}

This paper provides a description of individuals who had their inpatient psychiatric stay paid for by WSIB (at least partially) over an 11-year period. Future studies should examine impacts of the policy introduced in January 2018 to determine whether changes are observed in the characteristics and needs of individuals 
admitted to inpatient psychiatry for (or related to) work-related mental health injuries. Sex- and age-based analyses are also needed to further elucidate the relationships observed. Data linkage with WSIB claims would allow further understanding of the circumstances of the workplace injury, as well as potentially enable understanding of causal pathways between workplace injury and mental health.

\section{DATA AVAILABILITY STATEMENT}

The data analyzed in this study is subject to the following licenses/restrictions: The data are made available to interRAI Fellows for research use under an existing license agreement interRAI has with the Canadian Institute for Health Information; note that the agreement is for research only, not commercial use. Students working under the supervision of an interRAI Fellow can apply for free access to the data, but are subject to terms of use. As part of interRAI's agreement with the Canadian Institute for Health Information, the data may not be transmitted to third parties; therefore, the data used in this study cannot be made available to others. Those interested in using the data can apply directly to the Canadian Institute for Health Information for access. Requests to access these datasets should

\section{REFERENCES}

1. Nicholson PJ. Common mental disorders and work. Br Med Bull. (2018) 126:113-21. doi: 10.1093/bmb/ldy014

2. Mental Health Commission of Canada. Making the Case for Investing in Mental Health in Canada. Ottawa, ON: Mental Health Commission of Canada (2013). p. 30. Available online at: https://www.mentalhealthcommission.ca/ sites/default/files/2016-06/Investing_in_Mental_Health_FINAL_Version_ ENG.pdf (accessed February 22, 2021).

3. Steel Z, Marnane C, Iranpour C, Chey T, Jackson JW, Patel V, et al. The global prevalence of common mental disorders: a systematic review and meta-analysis 1980-2013. Int J Epidemiol. (2014) 43:47693. doi: 10.1093/ije/dyu038

4. Meredith WR. The Meredith Report (1913). Available online at: https://awcbc. org/wp-content/uploads/2013/12/meredith_report.pdf (accessed January 22, 2020).

5. Theorell T, Hammarstrom A, Aronsson G, Bendz LT, Grape T, Hogstedt C, et al. A systematic review including meta-analysis of work environment and depressive symptoms. BMC Public Health. (2015) 15:738. doi: 10.1186/s12889-015-1954-4

6. Workplace Safety Insurance Board. Operational Policy Manual - Chronic Mental Stress (2018). Available online at: https://www.wsib.ca/en/ operational-policy-manual/chronic-mental-stress (accessed January 22, 2020).

7. Dewa CA, Lesage A, Goering $P$, Caveen $M$. Nature and prevalence of mental illness in the workplace. Healthc Pap. (2004) 5:12-25. doi: 10.12927/hcpap..16820

8. Dewa CA, Goering P, Lin E, Paterson M. Depression-related short-term disability in an employed population. J Occup Environ Med. (2002) 44:62833. doi: 10.1097/00043764-200207000-00007

9. Canadian Institutes of Health Research, Natural Sciences and Engineering Research Council of Canada, and Social Sciences and Humanities Research Council of Canada. Tri-Council Policy Statement: Ethical Conduct for Research Involving Humans. (2018). Ottawa, ON: Government of Canada (2018). p. 231. Available online at: https://ethics.gc.ca/eng/policy-politique_tcps2eptc2_2018.html (Accessed January 22, 2020). be directed to https://www.cihi.ca/en/access-data-and-reports/ make-a-data-request.

\section{ETHICS STATEMENT}

Ethical review and approval was not required for the study on human participants in accordance with the local legislation and institutional requirements. The ethics committee waived the requirement of written informed consent for participation.

\section{AUTHOR CONTRIBUTIONS}

$\mathrm{MH}$ conducted the analyses of the data, with input from the LM and VK. MH drafted the initial version of the manuscript, with major writing contributions by the LM. VL finalized the manuscript. All authors contributed to the development of the study topic and design, and provided valuable and important feedback on the manuscript to its completion.

\section{ACKNOWLEDGMENTS}

The authors gratefully acknowledge the database management support from colleagues at the University of Waterloo.

10. Hirdes JP, Marhaba M, Smith TF, Clyburn L, Mitchell L, Lemick RA, et al. Development of the Resident Assessment Instrument-Mental Health (RAIMH). Hosp Q. (2000) 4:44-53. doi: 10.12927/hcq.2000.16756

11. Hirdes JP, Ljunggren G, Morris JN, Frijters DH, Finne Soveri H, Gray L, et al. Reliability of the interRAI suite of assessment instruments: a 12-country study of an integrated health information system. BMC Health Serv Res. (2008) 8:277. doi: 10.1186/1472-6963-8-277

12. Hirdes JP, van Everdingen C, Ferris J, Franco-Martin M, Fries BE, Heikkilä $\mathrm{J}$, et al. The interRAI suite of mental health assessment instruments: an integrated system for the continuum of care. Front Psychiatry. (2020) 10:926. doi: 10.3389/fpsyt.2019.00926

13. Martin L, Hirdes JP, Morris JN, Montague P, Rabinowitz T, Fries BE. Validating the Mental Health Assessment Protocols (MHAPs) in the Resident Assessment Instrument Mental Health (RAI-MH). J Psychiatr Ment Hlt. (2009) 16:646-53. doi: 10.1111/j.1365-2850.2009.01429.x

14. Morris JN, Fries BE, Morris SA. Scaling ADLs within the MDS. J Gerontol Ser A. (1999) 54:546-53. doi: 10.1093/gerona/54.11.M546

15. Perlman CM, Hirdes JP. The Aggressive Behavior Scale: a new scale to measure aggression based on the minimum data set. J Am Geriatr Soc. (2008) 56:2298-303. doi: 10.1111/j.1532-5415.2008.02048.x

16. Morris JN, Fries BE, Mehr DR, Hawes C, Phillips C, Mor $\mathrm{V}$, et al. MDS cognitive performance scale. J Gerontol. (1994) 49:174-82. doi: 10.1093/geronj/49.4.M174

17. Burrows A, Morris JN, Simon SE, Hirdes JP, Phillips C. Development of a minimum data set-based depression rating scale for use in nursing homes. Age Ageing. (2000) 29:165-72. doi: 10.1093/ageing/29.2.165

18. Fries BE, Simon SE, Morris JN, Flodstrom C, Bookstein FL. Pain in U.S. nursing homes: validating a pain scale for the minimum data set. Gerontologist. (2001) 41:173-9. doi: 10.1093/geront/41.2.173

19. Martin L, Hirdes JP. Mental health needs and service use in Ontario. Healthc Manage Forum. (2009) 22:40-6. doi: 10.1016/S0840-4704(10)60 291-8

20. Hirdes JP, Curtin-Telegdi N, Mathias K, Perlman CM, Saarela T, Kolbeinsson $\mathrm{H}$, et al. InterRAI Mental Health Clinical Assessment Protocols (CAPs) - For use with Community and Hospital-Based Mental Health Assessment Instruments. Washington DC: interRAI (2011). p. 214. 
21. Workplace Safety and Insurance Board: Report builder. WSIB by the numbers (2019). Available online at: http://www.divxy123.ca/ReportBuilder2018/ Pages/report_builder.php

22. Macpherson RA, Koehoorn M, Fan J, Quirke W, Amick BC, Kraut A, et al. Do differences in work disability duration between men and women vary by province in Canada? J Occup Rehabil. (2019) 29:5608. doi: 10.1007/s10926-018-9819-1

23. Busse JW, Ebrahim S, Heels-Ansdell D, Wang L, Couban R, Walter SD. Association of worker characteristics and early reimbursement for physical therapy, chiropractic and opioid prescriptions with workers' compensation claim duration, for cases of acute low back pain: an observational cohort study. BMJ Open. (2015) 5:e007836. doi: 10.1136/bmjopen-2015-007836

24. Helliwell JF, Huang H. New measures of the costs of unemployment: evidence from the subjective well-being of 3.3 million Americans. Econ Inq. (2014) 52:1485-502. doi: 10.1111/ecin.12093

25. Turner JB, Turner RJ. Physical disability, unemployment, and mental health. Rehabil Psychol. (2004) 49:241-9. doi: 10.1037/0090-5550.49.3.241

26. Shaw WS, Roelofs C, Punnett L. Work environment factors and prevention of opioid-related deaths. Am J Public Health. (2020) 110:1235-41. doi: 10.2105/AJPH.2020.305716

27. De Boer J, Lok A, Van't Verlaat E, Duivenvoorden HJ, Bakker AB, Smit BJ. Work-related critical incidents in hospital-based health care providers and the risk of post-traumatic stress symptoms, anxiety, and depression: a meta-analysis. Social Science \& Medicine. (2011) 72:31626. doi: 10.1016/j.socscimed.2011.05.009

28. Carnide N, Hogg-Johnson S, Côté P, Irvin E, Van Eerd D, Koehoorn M, et al. Early prescription opioid use for musculoskeletal disorders and work outcomes: a systematic review of the literature. Clin J Pain. (2017) 33:647658. doi: 10.1097/AJP.0000000000000452

29. Carnide N, Hogg-Johnson S, Koehoorn M, Furlan AD, Côté P. Relationship between early prescription dispensing patterns and work disability in a cohort of low back pain workers' compensation claimants: a historical cohort study. Occup Environ Med. (2019) 76:573-81. doi: 10.1136/oemed-2018-10 5626

30. Barrett K, Chang YP. Behavioral interventions targeting chronic pain, depression, and substance use disorder in primary care. J Nurs Scholarsh. (2016) 48:345-53. doi: 10.1111/jnu.1 2213

31. Law PCF, Too LS, Butterworth P, Witt K, Reavley N, Milner AJ. A systematic review on the effect of work-related stressors on mental health of young workers. Int Ach Occup Environ Health. (2020) 93:61122. doi: 10.1007/s00420-020-01516-7

Conflict of Interest: The authors declare that the research was conducted in the absence of any commercial or financial relationships that could be construed as a potential conflict of interest.

Copyright (c) 2021 Herring, Martin and Kristman. This is an open-access article distributed under the terms of the Creative Commons Attribution License (CC BY). The use, distribution or reproduction in other forums is permitted, provided the original author(s) and the copyright owner(s) are credited and that the original publication in this journal is cited, in accordance with accepted academic practice. No use, distribution or reproduction is permitted which does not comply with these terms. 